Pacific Journal of Mathematics

ON INTEGRO-DIFFERENTIAL EQUATIONS IN BANACH 


\title{
ON INTEGRO-DIFFERENTIAL EQUATIONS IN BANACH SPACES
}

\author{
JÜRG T. MARTI
}

The abstract integro-differential equation

$$
d u(t) / d t=A u(t)+\int_{0}^{t} B(t-s) u(s) d s+f(t)
$$

is studied, where $u(t)$ and $f(t)$ are functions of $[0, \infty)$ to a Banach space $\mathfrak{X}, A$ and $B(t)$ are linear operators on $\mathfrak{X}$ to itself, $A$ is closed with domain $\mathscr{D}(A)$ and $B(t)$ and $f(t)$ are strongly continuous on $[0, \infty)$. Let $A$ be the infinitesimal generator of a semi-group of linear operators of class $\left(C_{0}\right)$ and let $u(t) \in \mathscr{D}(A)$ on $[0, \infty)$, where $u(0)$ is a prescribed initial value. It is then shown that there exists a unique strongly continuously differentiable solution of both the homogeneous and inhomogeneous problem. By the method of successive approximations, absolutely convergent series expansions of the solutions are obtained. Further it is proved that the solution operator of the $\odot$-adjoint homogeneous problem equals the $\odot$-adjoint of the solution operator of the homogeneous equation.

It is well known [1] that for $B(t) \equiv 0$ and $u(0) \in \mathscr{D}(A)$ the formally simpler homogeneous linear differential equation

$$
d u(t) / d t=A u(t)
$$

has the unique solution $u(t)=T(t) u(0) \in \mathscr{D}(A)$, where on $[0, \infty) T(t)$ is the semi-group of class $\left(C_{0}\right)$ with infinitesimal generator $A$ and there exists positive real numbers $M$ and $\beta$ such that $\|T(t)\| \leqq M \exp (\beta t)$. If we restrict the adjoint problem of (2) to the $\odot$-adjoint Banach space $\mathfrak{X}^{\odot}=\overline{\mathscr{D}\left(A^{*}\right)}$, the solution of $d u^{\odot}(t) / d t=A^{\odot} u^{\odot}(t)$, with initial value $u^{\odot}(0) \in \mathscr{D}\left(A^{\odot}\right)$, is given by $T^{\odot}(t) u^{\odot}(0)$. Both $A^{\odot}$ and $T^{\odot}(t)$ are restrictions of $A^{*}$ and $T^{*}(t)$ to $\mathfrak{X}^{\odot} . T^{\odot}(t)$ is generated by $A^{\odot}$ and is likewise a strongly continuous semi-group on $[0, \infty)$. Both solutions $u(t)$ and $u^{\odot}(t)$ are strongly continuously differentiable on $[0, \infty)$.

The more general problem with time-dependent $A$,

$$
d u(t) / d t=A(t) u(t)
$$

when the initial value $u(0)$ is prescribed was investigated by Kato $[2,3]$. A special case of this,

$$
d u(t) / d t=A u(t)+B(t) u(t)
$$

with given initial value $u(0), u(t) \in \mathscr{D}(A)$ on $[0, \infty)$ and where $B(t)$ is 
a strongly continuously differentiable one-parameter family of bounded linear operators on $[0, \infty]$ has been treated by Phillips [4]. This differential equation has a unique solution $U(t) u(0)$ where $U(t)$ is on $[0, \infty)$ a strongly continuous family of linear transformations on $\mathfrak{X}$ to itself. Again $u(t)$ is strongly continuously differentiable in $[0, \infty)$. $U(t)$ can be represented by a series expansion $\sum_{n=0}^{\infty} S_{n}(t)$, absolutely convergent in the uniform operator topology, uniformly in each finite interval of $[0, \infty)$.

Dealing now with integro-differential equations of type (1) some properties of solutions of (2) or (4) remain valid for solutions of (1). Specifically we obtain:

(a) The homogeneous problem has for $u(0) \in \mathscr{D}(A)$ and $t \geqq 0$ the unique strongly continuously differentiable solution $u(t)=U(t) u(0) \epsilon$ $\mathscr{D}(A)$ where $U(t)$ is strongly continuous on $[0, \infty), U(0)=I,\|U(t)\| \leqq$ $M \exp \left(\left(\beta+M_{t}\right) t\right), M_{t}=M \int_{0}^{t}\|B(s)\| d s$ and $U(t)=\sum_{n=0}^{\infty} S_{n}(t)$,

$$
S_{0}(t)=T(t), S_{n}(t) x=\int_{0}^{t} T(t-s) d s \int_{0}^{s} B(s-\sigma) S_{n-1}(\sigma) x d \sigma, \quad x \in \mathfrak{X} .
$$

The series expansion converges likewise absolutely in the uniform operator topology, uniformly in each finite subinterval of $[0, \infty)$.

(b) The inhomogeneous problem has for $u(0) \in \mathscr{D}(A)$ and $t \geqq 0$ the unique strongly continuously differentiable solution $\mathscr{D}(A) \ni u(t)=$ $U(t) u(0)+\sum_{n=0}^{\infty} g_{n}(t)$,

$$
g_{0}(t)=\int_{0}^{t} T(t-s) f(s) d s \text { and } g_{n}(t)=\int_{0}^{t} T(t-s) d s \int_{0}^{s} B(s-\sigma) g_{n-1}(\sigma) d \sigma .
$$

The convergence of the sum is absolute and uniform in each finite interval of $[0, \infty)$ and $\|u(t)\| \leqq M\left(1+K_{t}\right) \exp \left(\left(\beta+M_{t}\right) t\right)\|u(0)\|$ where $K_{t}=\int_{0}^{t}\|f(s)\| d s$.

(c) The solution $u^{\odot}(t)$ of the ๑-adjoint problem

$$
d u^{\odot}(t) / d t=A^{\odot} u^{\odot}(t)+\int_{0}^{t} B^{\odot}(t-s) u^{\odot}(s) d s,
$$

$u^{\odot}(t) \in \mathscr{D}\left(A^{\odot}\right)$ on $[0, \infty)$ with given initial value $u^{\odot}(0)$ and $B^{\odot}(t)$ strongly continuous on $[0, \infty)$ has all the properties of the solution of (1) listed under (a) and we have $u^{\odot}(t)=U^{\odot}(t) u(0), U^{\odot}(t)$ being the ๑-adjoint operator of $U(t)$. 
2. Existence and uniqueness of a strong solution of the homogeneous problem (1). Let $A$ be a closed linear operator on a Banach space $\mathfrak{X}$ to itself with domain $\mathscr{D}(A)$ dense in $\mathfrak{X}$ and let $\mathfrak{F}(\mathfrak{X})$ be the Banach algebra of all bounded linear transformations on $\mathfrak{X}$ to itself. We choose $A$ such that the resolvent $R(\lambda, A)$ for $n=1,2, \cdots$ and some real numbers $M>0$ and $\beta \geqq 0$ satisfies

$$
\left\|R(\lambda, A)^{n}\right\| \leqq M(\lambda-\beta)^{-n} \text { for } \lambda>\beta .
$$

By the Hille-Yosida-Phillips theorem [4, Theorem 2.1] this implies that $A$ generates a semi-group of class $\left(C_{0}\right)$ of linear operators on the semi-module $[0, \infty)$ to $\mathfrak{F}(\mathfrak{X})$ :

$$
\begin{aligned}
& \text { (i) } T\left(t_{1}+t_{2}\right)=T\left(t_{1}\right) T\left(t_{2}\right), \quad t_{1}, t_{2} \in[0, \infty), \\
& \text { (ii) } T(0)=I, \\
& \text { (iii) } T(t) \text { is strongly continuous on }[0, \infty) \text { and } \\
& \text { (iv) }\|T(t)\| \leqq M \exp (\beta t) .
\end{aligned}
$$

For $t>0 T(t)$ and $A$ commute on $\mathscr{D}(A)$ [1, Theorem 10.3.3] and for $x \in \mathscr{D}(A) T(t) x$ is strongly continuously differentiable in $[0, \infty)$ and is the unique solution [1, Corollary to Theorem 23.8.1] of the differential equation $d T(t) x / d t=A T(t) x$ with initial condition $T(0) x=x$. Instead of this we first investigate the homogeneous integro-differential equation

$$
d U(t) x / d t=A U(t) x+\int_{0}^{t} B(t-s) U(s) x d s
$$

for $U(t) x \in \mathscr{D}(A), t \geqq 0$ where the initial condition is $U(0) x=x$. We take $B(t)$ as a strongly continuous family of operators on $[0, \infty)$ to $\mathfrak{F}(\mathfrak{X})$. We have now the following theorem:

THEOREM 1. Let $B(t)$ be a strongly continuous function of $[0, \infty)$ to $\left(\mathfrak{S}(\mathfrak{X})\right.$ with $M_{t}=M \int_{0}^{t}\|B(s)\| d s$. Then it exists a unique one-parameter family of bounded linear operators $U(t)$ on $[0, \infty)$ to $\mathfrak{F}(\mathfrak{X})$ satisfying

(i) $U(t)$ is strongly continuous on $[0, \infty)$.

(ii) For $x \in \mathscr{D}(A) U(t) x$ is strongly continuously differentiable in $[0, \infty)$ and

(iii) is the unique solution of the integro-differential equation (7) with

(iv) $U(0)=I$.

(v) $U(t)$ has the representation

$$
U(t)=\sum_{n=0}^{\infty} S_{n}(t)
$$


where $S_{0}(t)=T(t)$ and

$$
S_{n}(t)=\int_{0}^{t} T(t-s) d s \int_{0}^{s} B(s-\sigma) S_{n-1}(\sigma) x d \sigma .
$$

The series expansion converges absolutely in the uniform operator topology, uniformly in each finite subinterval of $[0, \infty)$ and

(vi) $\|U(t)\| \leqq M \exp \left(\left(\beta+M_{t}\right) t\right)$.

In order to prove the theorem we need the following lemma [4, Lemma 6.1]:

LEMma 2. Let $F(t)$ and $f(t)$ be strongly continuous functions on $[0, \infty)$ to $\mathfrak{F}(\mathfrak{X})$ and to $\mathfrak{X}$ respectively. Then $g(t)=\int_{0}^{t} F(t-s) f(s) d s$ exists in the strong topology and is itself strongly continuous on $[0, \infty)$ to $\mathfrak{X}$. If $f(t)$ is strongly continuously differentiable then so is $g(t)$ and with $g^{\prime}(t)=d g(t) / d t$ we have

$$
g^{\prime}(t)=F(t) f(0)+\int_{0}^{t} F(t-s) f^{\prime}(s) d s .
$$

First of all we prove the uniform and absolute convergence of sum (8) for $U(t)$. Since $B(t)$ is strongly continuous on $[0, \infty),\|B(t)\|$ is bounded and measurable in each finite interval of $[0, \infty)$. Hence $\int_{0}^{t}\|B(s)\| d s$ exists and we take

$$
M_{t}=M \int_{0}^{t}\|B(s)\| d s .
$$

We suppose that for some $n \geqq 0 S_{n}(t)$ is strongly continuous on $[0, \infty)$ and that

$$
\left\|S_{n}(t)\right\| \leqq M t^{n} M_{t}^{n} \exp (\beta t) / n ! .
$$

Applying twice Lemma 2 we see that $S_{n+1}(t)$ is also strongly continuous on $[0, \infty)$. Further an estimate for $S_{n+1}(t)$ shows that (10) holds likewise for $n+1$. Because $S_{0}(t)$ is strongly continuous and $\left\|S_{0}(t)\right\| \leqq$ $M \exp (\beta t)(10)$ is valid for each $n \geqq 0$, hence in every finite interval of $[0, \infty)$ expansion (8) is absolutely and uniformly convergent in the uniform operator topology that proves (v). (vi) follows immediately from (10) and (iv) holds since $S_{0}(0)=I$ and $S_{n}(0)=0, n>0$. Let

$$
\left.U_{n}(t)=\sum_{k=0}^{n} S_{k}: t\right) .
$$

For $n \rightarrow \infty U_{n}(t)$ converges to $U(t)$ in the uniform operator topology, likewise absolutely and uniformly in each finite interval of $[0, \infty)$. Statement (i) then follows from the strong continuity of $U_{n}(t)$. In 
order to prove (ii) we consider the sum

$$
W(t) x=\sum_{n=0}^{\infty} S_{n}^{\prime}(t) x, \quad x \in \mathscr{D}(A) .
$$

Since for $x \in \mathscr{D}(A) S_{0}(t) x$ is strongly continuously differentiable Lemma 2 together with the definition of $S_{n}(t)$ indicates the strong continuous differentiability of $S_{n}(t) x$ for all $n \geqq 0$. For $n>0$

$$
S_{n}(t) x=\int_{0}^{t} T(s) d s \int_{s}^{t} B(\sigma-s) S_{n-1}(t-\sigma) x d \sigma,
$$

hence

$$
S_{n}^{\prime}(t) x=\int_{0}^{t} T(s) B(t-s) S_{n-1}(0) x d s+\int_{0}^{t} T(t-s) d s \int_{0}^{s} B(s-\sigma) S_{n-1}^{\prime}(\sigma) x d \sigma .
$$

If $n=1$ one obtains, using $S_{0}^{\prime}(t) x=T(t) A x$,

$$
\left\|S_{1}^{\prime}(t) x\right\| \leqq M M_{t}(\|x\|+t\|A x\|) \exp (\beta t)
$$

and we get for $n \geqq 1$ inductively

$$
\left\|S_{n}^{\prime}(t)\right\| \leqq M t^{n-1} M_{t}^{n}(\|x\|+t\|A x\|) \exp (\beta t) /(n-1) ! .
$$

This shows that the series expansion (12) is absolutely and uniformly convergent in every finite interval of $[0, \infty)$. Therefore $W(t) x$ is strongly continuous on $[0, \infty)$ and, by $(11)$ and (12), for $x \in \mathscr{D}(A)$ $W(t) x$ is equal to the strong derivative $U^{\prime}(t) x$ of $U(t) x$. By (11) we have

$$
U_{n}(t) x=T(t) x+\int_{0}^{t} T(t-s) d s \int_{0}^{s} B(s-\sigma) U_{n-1}(\sigma) x d \sigma, \quad n>0 .
$$

Taking the strong limit for $n \rightarrow \infty$ on both sides we get an integral equation for $U(t) x, x \in \mathfrak{X}$ :

$$
U(t) x=T(t) x+\int_{0}^{t} T(t-s) d s \int_{0}^{s} B(s-\sigma) U(\sigma) x d \sigma .
$$

With the use of (13) and the definition of the infinitesimal generator $A$ of $T(t)$ on $\mathscr{D}(A)$ by the strong limit

$$
A x=\lim _{\delta \rightarrow 0+} \frac{1}{\delta}(T(\delta)-I) x,
$$

we obtain for $x \in \mathscr{D}(A)$ 


$$
\begin{aligned}
U^{\prime}(t) x-A T(t) x= & \lim _{\delta \rightarrow 0+} \frac{1}{\delta}\left[\int_{0}^{t+\delta} T(t+\delta-s) d s \int_{0}^{s} B(s-\sigma) U(\sigma) x d \sigma\right. \\
& \left.-\int_{0}^{t} T(t-s) d s \int_{0}^{s} B(s-\sigma) U(\sigma) x d \sigma\right] \\
= & \lim _{\delta \rightarrow 0+} \frac{1}{\delta}(T(\delta)-I)(U(t) x-T(t) x) \\
& +\int_{0}^{t} B(t-s) U(s) x d s .
\end{aligned}
$$

We know that $U^{\prime}(t) x-A T(t) x \in \mathfrak{X}$. Hence the last limit exists in the strong topology, by (14) $U(t) x-T(t) x \in \mathscr{D}(A)$ and so also $U(t) x \in \mathscr{D}(A)$ and we get the integro-differential equation for $x \in \mathscr{D}(A)$

$$
d U(t) x / d t=A U(t) x+\int_{0}^{t} B(t-s) U(s) x d s .
$$

In order to prove that for $x \in \mathscr{D}(A)$ the solution $u(t)=U(t) x$ is unique and thus (iii) of Theorem 1, we show that every nul solution, i.e., every strongly continuously differentiable solution $u(t)$ of (15) with initial value $u(0)=0$ vanishes for $t \geqq 0$. We take a $t_{0}>0$ such that $M_{t_{0}} \int_{0}^{t_{0}} \exp (\beta s) d s<1$ and assume $u(t)=0$ for $t \in\left[0, n t_{0}\right]$ and some integer $n \geqq 0$. Multiplying both sides of (15) by $T(\sigma-t)$ and integrating over $\left[n t_{0}, \sigma\right]$ where $n t_{0} \leqq \sigma \leqq(n+1) t_{0}$ we get

$$
u(\sigma)=\int_{n t_{0}}^{o} T(\sigma-t) d t \int_{0}^{t} B(t-s) u(s) d s .
$$

Let $C_{n}=\sup \left[\|u(t)\| ; t \in\left[n t_{0},(n+1) t_{0}\right]\right]$. Then for $t \in\left[n t_{0},(n+1) t_{0}\right]$

$$
\|u(t)\| \leqq C_{n} M \int_{n t_{0}}^{t} \exp (\beta(t-\sigma)) d \sigma \int_{n t_{0}}^{\sigma}\|B(\sigma-s)\| d s
$$

and

$$
C_{n} \leqq C_{n} M_{t_{0}} \int_{0}^{t_{0}} \exp (\beta s) d s
$$

so that $C_{n}=0$ and $u(t)=0$ for $t \in\left[0,(n+1) t_{0}\right]$. Since $u(0)=0$ it follows at once by recursion that $u(t)=0$ on $[0, \infty)$.

\section{The inhomogeneous problem.}

THEOREM 3. Let $B(t)$ be a strongly continuous function of $[0, \infty)$ to $\left(\mathfrak{S}\left(\mathfrak{X )}\right.\right.$ with $M_{t}=M \int_{0}^{t}\|B(s)\| d s$ and let $f(t)$ be a strongly 
continuously differentiable function of $[0, \infty)$ to $\mathfrak{X}$ with $K_{t}=$ $\int_{0}^{t}\|f(s)\| d s$. Then the inhomogeneous problem

$$
d u(t) / d t=A u(t)+\int_{0}^{t} B(t-s) u(s) d s+f(s)
$$

has for each $u(0) \in \mathscr{D}(A)$ a unique continuously differentiable solution $u(t)$ on $[0, \infty)$ to $\mathscr{D}(A)$. $u(t)$ has the representation

$$
u(t)=U(t) u(0)+\sum_{n=0}^{\infty} g_{n}(t)
$$

where $U(t) u(0)$ is the solution of the homogeneous problem described in Theorem 1, $g_{0}(t)=\int_{0}^{t} T(t-s) f(s) d s$ and for $n>0$

$$
g_{n}(t)=\int_{0}^{t} T(t-s) d s \int_{0}^{s} B(s-\sigma) g_{n-1}(\sigma) d \sigma
$$

The sum converges absolutely and uniformly in each finite interval of $[0, \infty)$. Further $\|u(t)\| \leqq M\left(1+K_{t}\right) \exp \left(\left(\beta+M_{t}\right) t\right)\|u(0)\|$.

Proof. Through repeated application of Lemma 2 it follows inductively that for $n \geqq 0$ the $g_{n}(t)$ are strongly continuously differentiable. We obtain in an analogous way as in the proof of Theorem 1

$$
\begin{aligned}
& g_{0}^{\prime}(t)=T(t) f(0)+\int_{0}^{t} T(t-s) f^{\prime}(s) d s \\
& g_{n}^{\prime}(t)=\int_{0}^{t} T(t-s) d s \int_{0}^{s} B(s-\sigma) g_{n-1}^{\prime}(\sigma) d \sigma
\end{aligned}
$$

and for $n \geqq 0$

$$
\begin{aligned}
& \left\|g_{n}(t)\right\| \leqq M t^{n} K_{t} M_{t}^{n} \exp (\beta t) / n ! \\
& \left\|g_{n}^{\prime}(t)\right\| \leqq M t^{n} L_{t} M_{t}^{n} \exp (\beta t) / n !
\end{aligned}
$$

where $L_{t}=\|f(0)\|+\int_{0}^{t}\left\|f^{\prime}(s)\right\| d s$. This shows that the sum $\sum_{n=0}^{\infty} g_{n}(t)$ is a strongly continuously differentiable function $g(t)$ of $[0, \infty)$ to $\mathfrak{X}$, where $g(0)=0$. Due to the uniform convergence of $\sum_{n=0}^{\infty} g_{n}(s)$ in $[0, t]$ we get from the definition of $g_{n}(t)$ an integral equation

$$
\begin{aligned}
g(t) & =g_{0}(t)+\lim _{n \rightarrow \infty} \int_{0}^{t} T(t-s) d s \int_{0}^{s} B(s-\sigma) \sum_{j=0}^{n} g_{j}(\sigma) d \sigma \\
& =\int_{0}^{t} T(t-s)\left[f(s)+\int_{0}^{s} B(s-\sigma) g(\sigma) d \sigma\right] d s .
\end{aligned}
$$

Similarly, to the manner in which we derived the integro-differential 
equation (15) from the integral equation (13), we obtain

$$
g^{\prime}(t)=f(t)+\int_{0}^{t} B(t-s) g(s) d s+\lim _{\delta \rightarrow 0} \frac{1}{\delta}(T(\delta)-I) g(t) .
$$

Since for fixed $t$ the limit exists in the strong topology we have $g(t) \in \mathscr{D}(A)$ and the last term on the right side of (17) equals $A g(t)$. Hence $g(t)$ is a particular solution of (16) and by Theorem $1 u(t)=$ $U(t) u(0)+g(t)$ is a solution of (16) where $u(0) \in \mathscr{D}(A)$ implies $u(t) \in \mathscr{D}(A), t \geqq 0$.

To pove the uniqueness of this solution we suppose to have two solutions of (16) with same initial value. Then the difference of these two solutions is a solution of the corresponding homogeneous equation with initial value equal to zero. Due to the uniqueness of the solution of the homogeneous problem (Theorem 1) this nul solution vanishes.

4. The adjoint problem. Since $A$ is a linear transformation with domain $\mathscr{D}(A)$ dense in $\mathfrak{X}$ the adjoint $A^{*}$ of $A$ is a closed linear transformation on $\mathscr{D}\left(A^{*}\right) \subset \mathfrak{X}^{*}$ to $\mathfrak{X}^{*}$. But in general $\mathscr{D}\left(A^{*}\right)$ is not dense in $\mathfrak{X}^{*}$ so that $A^{*}$ is not necessarily the infinitesimal generator of a strongly continuous semi-group in $\mathfrak{X}^{*}$. Therefore we restrict the treatment of the adjoint problem of (7) to the ๑-adjoint space $\mathfrak{X}^{\odot}$ of $\mathfrak{X}$, defined by $\mathfrak{X}^{\odot}=\overline{\mathscr{D}\left(A^{*}\right)}$. In case $A \in \mathfrak{F}(\mathfrak{X})$ or if $\mathfrak{X}$ is reflexive we have $\mathfrak{X}^{\odot}=\mathfrak{X}^{*}$, else $\mathfrak{X}^{\odot}$ may be a proper subset of $\mathfrak{X}^{*}$. Given a linear operator $Q$ on $\mathfrak{X}$ to itself with dense domain we denote by $Q^{\circ}$ the restriction of $Q^{*}$ with domain $\mathscr{D}\left(Q^{\odot}\right)=\left[x^{*} ; x^{*} \in \mathscr{D}\left(Q^{*}\right) \cap \mathfrak{X}^{\odot}, Q^{*} x^{*} \in \mathfrak{X}^{\odot}\right]$. Let $T^{*}(t)$ then be the adjoint transformation of $T(t)$ and $T^{\odot}(t)$ the restriction of $T^{*}(t)$ to $\mathfrak{X}^{\odot}$ in the sense described above. Then by [ 1 , Theorem and Corollary to Theorem 14.4.1] $T^{\odot}(t) \in \mathfrak{E}(\mathfrak{X} \odot)$ is a semi-group of class $\left(C_{0}\right)$ and its infinitesimal generator is $A^{\circ}$. Clearly we have $\left\|T^{\odot}(t)\right\| \leqq\|T(t)\| \leqq M \exp (\beta t)$.

We suppose the $\odot$-adjoint $B^{\odot}(t)$ of $B(t)$ to be a linear operator on $[0, \infty)$ to $\left[\mathfrak{F}\left(\mathfrak{X}^{\odot}\right)\right.$, likewise strongly continuous (this is the case if $B(t)$ is uniformly continuous) and state the ๑-adjoint problem

$$
d V(t) x^{\odot} / d t=A^{\odot} V(t) x^{\odot}+\int_{0}^{t} B^{\odot}(t-s) V(s) x^{\odot} d s
$$

for $V(t) x^{\odot} \in \mathscr{D}\left(A^{\odot}\right), t \geqq 0$ and the initial condition $V(0) x^{\odot}=x^{\odot}$. Then Theorem 1 applies for $B(t), U(t), T(t), A, \mathfrak{X}$ and $x$ replaced by $B^{\odot}(t), V(t)$, $T^{\odot}(t), A^{\odot}, \mathfrak{X}^{\odot}$ and $x^{\odot}$ respectively and we have the following

THEOREM 4. Let $B^{\odot}(t)$ be a strongly continuous lineàr transfor- 
mation on $[0, \infty)$ to $\mathfrak{F}\left(\mathfrak{X}^{\circ}\right)$. Then the solution $V(t)$ of the $\odot$-adjoint problem (18) is identical with the ๑-adjoint $U^{\odot}(t)$ of the solution $U(t)$ of problem (7).

Proof. By Theorem $1 V(t)$ has the representation

$$
V(t)=\sum_{n=0}^{\infty} V_{n}(t)
$$

where $V_{0}(t)=T^{\odot}(t)$, for $x^{\odot} \in \mathfrak{X} \odot$ and $n>0$

$$
V_{n}(t) x^{\odot}=\int_{0}^{t} T^{\odot}(t-s) d s \int_{0}^{s} B^{\odot}(s-\sigma) V_{n-1}(\sigma) x^{\odot} d \sigma
$$

is strongly continuous on $[0, \infty)$ and the expansion converges absolutely in the uniform operator topology.

We now prove that $V_{n}(t)$ is identical to the $\odot$-adjoint $S_{n}^{\odot}(t)$ of the term $S_{n}(t)$ occurring in (8). This is trivial for $n=0$. For $n>0$ $S_{n}(t)$ is bounded so that $S_{n}^{*}(t)$ exists as a bounded linear operator on $\mathfrak{X}^{*}$ to itself, for each $x \in \mathfrak{X}$ and $x^{*} \in \mathfrak{X}^{*}$ defined by

$$
\begin{aligned}
{\left[S_{n}^{*}(t) x^{*}\right] x } & =x^{*}\left[S_{n}(t) x\right] \\
& =x^{*}\left[\int_{0}^{t} \cdots \int_{0}^{t} T\left(t-s_{1}\right) \prod_{i=1}^{n}\left\{B\left(s_{\imath}-t_{i}\right) T\left(t_{i}-s_{i+1}\right) d s_{i} d t_{i}\right\} x\right]
\end{aligned}
$$

where we take $s_{n+1}=0, t_{0}=t$ and $B(t)=T(t)=0$ for $t<0$. Since $\left\|x^{*}\right\|<\infty$ we have

$$
\begin{aligned}
& {\left[S_{n}^{*}(t) x^{*}\right] x=\int_{0}^{t} \cdots \int_{0}^{t} x^{*}\left[T\left(t-s_{1}\right) \prod_{i=1}^{n}\left\{B\left(s_{i}-t_{i}\right) T\left(t_{i}-s_{i+1}\right) d s_{i} d t_{i}\right\} x\right]} \\
& \quad=\int_{0}^{t} \cdots \int_{0}^{t}\left[T^{*}\left(t_{n}\right) \prod_{i=1}^{n}\left\{B^{*}\left(S_{n-i+1}-t_{n-i+1}\right) T^{*}\left(t_{n-i}-s_{n-i+1}\right) d s_{i} d t_{i}\right\} x^{*}\right] x .
\end{aligned}
$$

Substituting $t_{i}^{\prime}=t-s_{n-i+1}, s_{i}^{\prime}=t-t_{n-i+1}, i=1, \cdots, n$ we get, applying the theorem of Fubini and suppressing apostrophes

$$
\left[S_{n}^{*}(t) x^{*}\right] x=\int_{0}^{t} \cdots \int_{0}^{t}\left[T^{*}\left(t-s_{1}\right) \prod_{i=1}^{n}\left\{B^{*}\left(s_{i}-t_{i}\right) T^{*}\left(t_{i}-s_{i+1}\right) d s_{i} d t_{i}\right\} x^{*}\right] x .
$$

For $x^{*}=x^{\odot} \in \mathfrak{X}^{\odot}$ it follows since $T^{\odot}(t)$ and $B^{\odot}(t)$ are strongly continuous and elements of $\left(\mathfrak{S}\left(\mathfrak{X}^{\circ}\right)\right.$

$$
\left[S_{n}^{*}(t) x^{\odot}\right] x=\left[V_{n}(t) x^{\odot}\right] x,
$$

therefore $S_{n}^{*}(t) x^{\odot} \in \mathfrak{X}^{\odot}$ and we have for each $x^{\odot} \in \mathfrak{X}^{\odot}$

$$
V_{n}(t) x^{\odot}=S_{n}^{\odot}(t) x^{\odot} \text {. }
$$

For $Q \in \mathfrak{F}(\mathfrak{X})$ the transformation $Q \rightarrow Q^{*}$ is an isometry of $\mathfrak{F}(\mathfrak{X})$ into 
$\mathfrak{F}\left(\mathfrak{X}^{*}\right)$. Due to the absolute convergence in the uniform operator topology of expansion (8) we then have

$$
U^{*}(t)=\left[\lim _{N \rightarrow \infty} \sum_{n=0}^{N} S_{n}(t)\right]^{*}=\sum_{n=0}^{\infty} S_{n}^{*}(t) .
$$

Thus for $x^{\odot} \in \mathfrak{X}^{\odot}$ we get

$$
U^{*}(t) x^{\odot}=\sum_{n=0}^{\infty} S_{n}^{*}(t) x^{\odot}=\sum_{n=0}^{\infty} V_{n}(t) x^{\odot}=V(t) x^{\odot},
$$

hence $U^{*}(t) x^{\odot} \in \mathfrak{X}^{\odot}$ and

$$
V(t)=U^{\odot}(t)
$$

Acknowledgments. The author is indebted to Professor Dr. A. Pfluger at the Department of Mathematics of the Swiss Federal Institute of Technology for many valuable discussions.

\section{REFERENCES}

1. E. Hille and R. S. Phillips, Functional analysis and semi-groups, Amer. Math. Soc. Colloquium Publ. Vol. 31, rev. ed., 1957.

2. T. Kato, On linear differential equations in Banach spaces, Comm. on Pure and Appl. Math. 9 (1956), 479-486.

3. Integration of the equation of evolution in a Banach space, J. Math. Soc. Japan 5 (1953), 208-234.

4. R. S. Phillips, Perturbation theory for semi-groups of linear operators, Trans. Amer. Math. Soc. 74 (1953), 199-221.

Received August 2, 1965.

EIDG. Instiut F. REAKTORFORSCHUNG, 5303 WÜrENLINGEN

SWITZERLAND 


\section{PACIFIC JOURNAL OF MATHEMATICS}

H. SAMELSON

Stanford University

Stanford, California

J. P. JaNs

University of Washington

Seattle, Washington 98105

\section{EDITORS}

J. DugundJI

University of Southern California Los Angeles, California 90007

RICHARD ARENS

University of California

Los Angeles, California 90024

\section{ASSOCIATE EDITORS}

E. F. BECKENBACH

B. H. NEUMANN

F. WOLF

K. YosidA

\section{SUPPORTING INSTITUTIONS}

UNIVERSITY OF BRITISH COLUMBIA

CALIFORNIA INSTITUTE OF TECHNOLOGY

UNIVERSITY OF CALIFORNIA

MONTANA STATE UNIVERSITY

UNIVERSITY OF NEVADA

NEW MEXICO STATE UNIVERSITY

OREGON STATE UNIVERSITY

UNIVERSITY OF OREGON

OSAKA UNIVERSITY

UNIVERSITY OF SOUTHERN CALIFORNIA
STANFORD UNIVERSITY

UNIVERSITY OF TOKYO

UNIVERSITY OF UTAH

WASHINGTON STATE UNIVERSITY

UNIVERSITY OF WASHINGTON

AMERICAN MATHEMATICAL SOCIETY CHEVRON RESEARCH CORPORATION TRW SYSTEMS

NAVAL ORDNANCE TEST STATION 


\section{Pacific Journal of Mathematics}

\section{Vol. 20, No. $1 \quad$ September, 1967}

Leonard Daniel Baumert, Extreme copositive quadratic forms. II ........ 1

Edward Lee Bethel, A note on continuous collections of disjoint

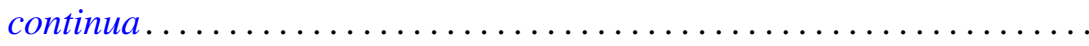

Delmar L. Boyer and Adolf G. Mader, A representation theorem for abelian groups with no elements of infinite p-height ...................

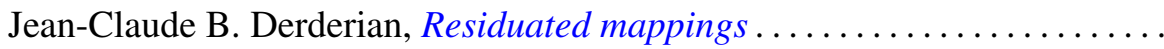

Burton I. Fein, Representations of direct products of finite groups ......... 45

John Brady Garnett, A topological characterization of Gleason parts.......

Herbert Meyer Kamowitz, On operators whose spectrum lies on a circle or

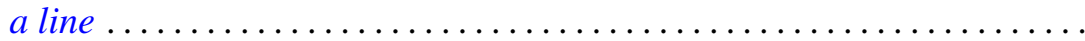

Ignacy I. Kotlarski, On characterizing the gamma and the normal distribution ........................................

Yu-Lee Lee, Topologies with the same class of homeomorphisms ..........

Moshe Mangad, Asymptotic expansions of Fourier transforms and discrete polyharmonic Green's functions ...........................

Jürg Thomas Marti, On integro-differential equations in Banach spaces ....

Walter Philipp, Some metrical theorems in number theory.............. 109

Maxwell Alexander Rosenlicht, Another proof of a theorem on rational

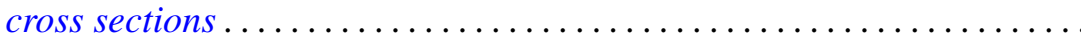

Kenneth Allen Ross and Karl Robert Stromberg, Jessen's theorem on Riemann sums for locally compact groups

Stephen Simons, A theorem on lattice ordered groups, results of Ptak, Namioka and Banach, and a front-ended proof of Lebesgue's theorem...

Morton Lincoln Slater, On the equation $\varphi(x)=\int_{x} x+1 K(\xi) f[\varphi(\xi)] d \xi \ldots$ 155 Arthur William John Stoddart, Existence of optimal controls .. 167 Burnett Roland Toskey, A system of canonical forms for rings on a direct sum of two infinite cyclic groups ....................

Jerry Eugene Vaughan, A modification of Morita's characterization of dimension 